(C) Revista de Matemática: Teoría y AplicaCiones 2019 26(2) : 197-214

CIMPA - UCR ISSN: 1409-2433 (PRINT), 2215-3373 (ONLINE)

DOI: https://doi.org/10.15517/rmta.v26i2.38315

\title{
A BRIEF SURVEY OF HIGGS BUNDLES
}

\section{UN ESTUDIO CONCISO DE FIBRADOS DE HIGGS}

\author{
RONALD A. ZÚÑIGA-ROJAS*
}

Received: 9/May/2018; Revised: 5/Jun/2019;

Accepted: 6/Jun/2019

\begin{abstract}
Revista de Matemática: Teoría y Aplicaciones is licensed under a Creative Commons Reconocimiento-NoComercial-Compartirigual 4.0 International License.

Creado a partir de la obra en http://www.revistas.ucr.ac.cr/index.php/matematica
\end{abstract}

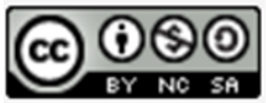

*Universidad de Costa Rica, Escuela de Matemática, Centro de Investigaciones Matemáticas y Metamatemáticas CIMM, San José, Costa Rica. E-Mail: ronald.zunigarojas@ucr.ac.cr 


\begin{abstract}
Considering a compact Riemann surface of genus greater or equal than two, a Higgs bundle is a pair composed of a holomorphic bundle over the Riemann surface, joint with an auxiliar vector field, so-called Higgs field. This theory started around thirty years ago, with Hitchin's work, when he reduced the self-duality equations from dimension four to dimension two, and so, studied those equations over Riemann surfaces. Hitchin baptized those fields as Higgs fields because in the context of physics and gauge theory, they describe similar particles to those described by the Higgs bosson. Later, Simpson used the name Higgs bundle for a holomorphic bundle together with a Higgs field. Today, Higgs bundles are the subject of research in several areas such as non-abelian Hodge theory, Langlands, mirror symmetry, integrable systems, quantum field theory (QFT), among others. The main purposes here are to introduce these objects, and to present a brief but complete construction of the moduli space of Higgs bundles.
\end{abstract}

Keywords: Higgs bundles; Hodge bundles; moduli spaces; stable triples; vector bundles.

\title{
Resumen
}

Considerando una superficie compacta de Riemann de género mayor o igual que dos, un fibrado de Higgs es un par compuesto por un fibrado holomorfo sobre la superficie de Riemann, junto con un campo vectorial auxiliar, llamado campo de Higgs. Esta teoría inició hace unos treinta años, con el trabajo de Hitchin, cuando él reduce las ecuaciones de autodualidad de dimensión cuatro a dimensión dos, y así, estudiar esas ecuaciones sobre superficies de Riemann. Hitchin bautizó esos campos como campos de Higgs pues en el contexto de la física y de la teoría de gauge, describen partículas similares a las descritas por el bozón de Higgs. Más tarde, Simpson usó el nombre fibrado de Higgs para un fibrado holomorfo junto con un campo de Higgs. Hoy, los fibrados de Higgs son objeto de investigación en varias áreas tales como la teoría de Hodge no abeliana, Langlands, simetría de espejo, sistemas integrables, teoría cuántica de campos (QFT), entre otros. Los propósitos principales aquí son introducir estos objetos y presentar una breve pero completa construcción del espacio móduli de los fibrados de Higgs y algunas de sus estratificaciones.

Palabras clave: fibrados de Higgs; fibrados de Hodge; espacios móduli; triples estables; fibrados vectoriales.

Mathematics Subject Classification: Primary 14H60, Secondaries 14D07, 55Q52. 


\section{Introduction}

Nineteen years ago, the Clay Institute of Cambridge, Massachusets, presented seven millenium challenging problems. Yang-Mills and Mass Gap is one of those challenging unsolved problems. Newton's classical mechanics laws stand to planets and celestial bodies as Quantum laws stand to elementary particles. Sixty five years ago, C. N. Yang and R. L. Mills [18] introduced a remarkable framework to describe particles using structures that occur in geometry. Quantum Yang-Mills theory is nowadays the foundation of elementary particle physics theory and its predictions have been tested at many laboratories; nevertheless, its mathematical foundations are still a mystery. Yang-Mills theory depends on a quantum mechanical property, the mass gap: the quantum particles have positive masses, even though the classical waves travel at the speed of light. Establishing the existence of the Yang-Mills theory and a mass gap will require the introduction of fundamental new ideas both in physics and in mathematics. Thirty two years ago, Hitchin [9] reduced Yang-Mills self-duality equations from $\mathbb{R}^{4}$ to $\mathbb{R}^{2}$ imposing invariance translation in two directions, and considering an auxiliar vector field, so-called Higgs field. Hitchin's reduction of Yang-Mills self-duality equations in $\mathbb{R}^{2}$ have the important property of conformal invariance, which allows them to be defined on Riemann surfaces.

Let $\Sigma=\Sigma_{g}$ be a compact (closed and connected) Riemann surface of genus $g \geqslant 2$. Let $K=K_{\Sigma}=T^{*} \Sigma$ be the canonical line bundle over $\Sigma$ (its cotangent bundle).

Remark 1. Algebraically, $\Sigma_{g}$ is also a complete irreducible nonsingular algebraic curve over $\mathbb{C}$ :

$$
\operatorname{dim}_{\mathbb{C}}\left(\Sigma_{g}\right)=1
$$

The paper is organized as follows: in Section 2 we recall some preliminary facts about differential geometry, specifically about smooth vector bundles and holomorphic vector bundles; in Section 3, we present the gauge group action over the set of connections and the induced action over the holomorphic structures; in Section 4, we define the moduli space of Higgs bundles, and we present the two principal Hitchin constructions for rank two: the differential geometry flavor, and the algebraic geometry flavor in 4.2; in 4.3 we present Simpsons contribution to higher rank; in Section 5, we recall some basic facts about stratifications of the moduli space of Higgs bundles: Shatz stratification in 5.1 and Białynicki-Birula stratification in 5.2; in Section 6, we present our most recent results, those related to stratifications published in [5], and those related to homotopy published in [20]. 


\section{Preliminary definitions}

We shall recall some basic definitions we will need, like bundles and some of their invariants such as rank, degree and slope. We also present the definition of stability.

Definition 1. For any smooth vector bundle $\mathcal{E} \rightarrow \Sigma$, we denote the rank of $\mathcal{E}$ by $\operatorname{rk}(\mathcal{E})=r$ and the degree of $\mathcal{E}$ by $\operatorname{deg}(\mathcal{E})=d$. Then, for any smooth complex bundle $\mathcal{E} \rightarrow \Sigma$ the slope is defined to be

$$
\mu(\mathcal{E}):=\frac{\operatorname{deg}(\mathcal{E})}{\operatorname{rk}(\mathcal{E})}=\frac{d}{r} .
$$

Definition 2. A smooth vector bundle $\mathcal{E} \rightarrow \Sigma$ is called semistable if $\mu(\mathcal{F}) \leqslant$ $\mu(\mathcal{E})$ for any $\mathcal{F}$ such that $0 \subsetneq \mathcal{F} \subseteq \mathcal{E}$. Similarly, a vector bundle $\mathcal{E} \rightarrow \Sigma$ is called stable if $\mu(\mathcal{F})<\mu(\mathcal{E})$ for any nonzero proper subbundle $0 \subsetneq \mathcal{F} \subsetneq \mathcal{E}$. Finally, $\mathcal{E} \rightarrow \Sigma$ is called polystable if it is the direct sum of stable subbundles, all of the same slope.

Let $\mathcal{E} \rightarrow \Sigma$ be a complex smooth vector bundle with a hermitian metric on it.

Definition 3. A connection $d_{A}$ on $\mathcal{E}$ is a differential operator

$$
d_{A}: \Omega^{0}(\Sigma, \mathcal{E}) \rightarrow \Omega^{1}(\Sigma, \mathcal{E})
$$

such that

$$
d_{A}(f s)=d f \otimes s+f d_{A} s
$$

for any function $f \in C^{\infty}(\Sigma)$ and any section $s \in \Omega^{0}(\Sigma, \mathcal{E})$ where $\Omega^{n}(\Sigma, \mathcal{E})$ is the set of smooth $n$-forms of $\Sigma$ with values in $\mathcal{E}$. Locally:

$$
d_{A}=d+A=d+C d z+B d \bar{z}
$$

where $A$ is a matrix of 1-forms: $A_{i j} \in \Omega^{1}(\Sigma, \mathcal{E})$, and $B, C$ are matrix valued functions depending on the hermitian metric on $\mathcal{E}$.

Definition 4. When a connection $d_{A}$ is compatible with the hermitian metric on $\mathcal{E}$,

$$
\text { i.e. } \quad d\langle s, t\rangle=\left\langle d_{A} s, t\right\rangle+\left\langle s, d_{A} t\right\rangle,
$$

for the hermitian inner product $\langle\cdot, \cdot\rangle$ and for $s, t$ any couple of sections of $\mathcal{E}, d_{A}$ is a unitary connection. Denote $\mathcal{A}(\mathcal{E})$ as the space of unitary connections on $\mathcal{E}$, for a smooth bundle $\mathcal{E} \rightarrow \Sigma$. 
Remark 2. Some authors call the matrix $A$ as a connection and call $d_{A}=$ $d+A$ as its correspoding covariant derivative. We abuse notation and will not distinguish between them.

Definition 5. The fundamental invariant of a connection is its curvature:

$$
F_{A}:=d_{A}^{2}=d_{A} \circ d_{A}: \Omega^{0}(\Sigma, \mathcal{E}) \rightarrow \Omega^{2}(\Sigma, \mathcal{E}),
$$

where we are extending $d_{A}$ to $n$-forms in $\Omega^{n}(\Sigma, \mathcal{E})$ in the obvious way. Locally:

$$
F_{A}=d A+A^{2} .
$$

$F_{A}$ is $C^{\infty}(\Sigma, \mathcal{E})$-linear and can be considered as a 2-form on $\Sigma$ with values in $\operatorname{End}(\mathcal{E}): F_{A} \in \Omega^{2}(\Sigma, \operatorname{End}(\mathcal{E}))$, or locally as a matrix-valued 2-form.

Definition 6. If the curvature vanishes $F_{A}=0$, we say that the connection $d_{A}$ is flat. A flat connection gives a family of constant transition functions for $\mathcal{E}$, which in turn defines a representation of the fundamental group $\pi_{1}(\Sigma)$ into $G L_{r}(\mathbb{C})$, and the image is in $U(r)$ if $d_{A}$ is unitary. Besides, from Chern-Weil theory, if $F_{A}=0$, then $\operatorname{deg}(\mathcal{E})=0$.

\section{Gauge group action}

The gauge theory arises first in the context of physics, specifically in general relativity and classical electromagnetism. Here, we present the mathematical formalism.

Definition 7. A gauge transformation is an automorphism of $\mathcal{E}$. Locally, a gauge transformation $g \in A u t(\mathcal{E})$ is a $C^{\infty}$-function with values in $G L_{r}(\mathbb{C})$. A gauge transformation $g$ is unitary if $g$ preserves the hermitian inner product.

We denote by $\mathcal{G}$ the group of unitary gauge transformations. This gauge group $\mathcal{G}$ acts on $\mathcal{A}(\mathcal{E})$ by conjugation:

$$
g \cdot d_{A}=g^{-1} d_{A} g, \quad \forall g \in \mathcal{G} \text { and for } d_{A} \in \mathcal{A}(\mathcal{E})
$$

Remark 3. Note that conjugation by a unitary gauge transformation takes a unitary connection to a unitary connection. We denote by $\overline{\mathcal{G}}$ the quotient of $\mathcal{G}$ by the central subgroup $U(1)$, and by $\mathcal{G}^{\mathbb{C}}$ the complex gauge group. Besides, we denote by $B \mathcal{G}$ and by $B \overline{\mathcal{G}}$ the classifying spaces of $\mathcal{G}$ and $\overline{\mathcal{G}}$ respectively. There is a homotopical equivalence: $\mathcal{G} \simeq \mathcal{G}^{\mathbb{C}}$.

Rev.Mate.Teor.Aplic. (ISSN print: 1409-2433; online: 2215-3373) Vol. 26(2): 197-214, Jul-Dec 2019 
Definition 8. A holomorphic structure on $\mathcal{E}$ is a differential operator:

$$
\bar{\partial}_{A}: \Omega^{0}(\Sigma, \mathcal{E}) \rightarrow \Omega^{0,1}(\Sigma, \mathcal{E}),
$$

such that $\bar{\partial}_{A}$ satisfies Liebniz rule:

$$
\bar{\partial}_{A}(f s)=\bar{\partial} f \otimes s+f \bar{\partial}_{A} s
$$

and the integrability condition, which is precisely vanishing of the curvature

$$
F_{A}=0 .
$$

Here, $\bar{\partial} f=\frac{\partial f}{\partial \bar{z}} d \bar{z}$, and $\Omega^{p, q}(\Sigma, \mathcal{E})$ is the space of smooth $(p, q)$-forms with values in $\mathcal{E}$. Locally:

$$
\bar{\partial}_{A}=\bar{\partial}+A^{0,1} d \bar{z}
$$

where $A^{0,1}$ is a matrix valued function. The reader may consult the details in $[10,11,12]$.

Remark 4. Denote the space of holomorphic structures on $\mathcal{E}$ by $\mathcal{A}^{0,1}(\mathcal{E})$, and consider the collection $\mathcal{A}^{0,1}(r, d)=\left\{\mathcal{A}^{0,1}(\mathcal{E}): \operatorname{rk}(\mathcal{E})=r\right.$ and $\left.\operatorname{deg}(\mathcal{E})=d\right\}$. Here, the subcollections

$$
\begin{aligned}
& \mathcal{A}_{s s}^{0,1}(r, d)=\left\{\mathcal{A}^{0,1}(\mathcal{E}): \mathcal{E} \quad \text { is semistable }\right\} \subseteq \mathcal{A}^{0,1}(r, d), \\
& \mathcal{A}_{s}^{0,1}(r, d)=\left\{\mathcal{A}^{0,1}(\mathcal{E}): \mathcal{E} \quad \text { is stable } \quad\right\} \subseteq \mathcal{A}^{0,1}(r, d), \\
& \mathcal{A}_{p s}^{0,1}(r, d)=\left\{\mathcal{A}^{0,1}(\mathcal{E}): \mathcal{E} \quad \text { is polystable }\right\} \subseteq \mathcal{A}^{0,1}(r, d),
\end{aligned}
$$

will be of particular interest for us.

\section{Moduli space of Higgs bundles}

A central feature of Higgs bundles, is that they come in collections parametrized by the points of a quasi-projective variety: the Moduli Space of Higgs Bundles.

\subsection{Moduli space of vector bundles}

The starting point to classify these collections, is the Moduli Space of Vector bundles. Using Mumford's Geometric Invariant Theory ${ }^{1}$ (GIT), first Narasimhan and Seshadri [14], and then Atiyah and Bott [1], build and charaterize this family of bundles by Morse theory and by their stability. Both classifications are equivalent.

\footnotetext{
${ }^{1}$ For details on GIT, see Mumford, Fogarty and Kirwan [13].
} 
We use the one from stability here:

Definition 9. Define the moduli space of (polystable) vector bundles $\mathcal{E} \rightarrow \Sigma$ as the quotient

$$
\mathcal{N}(r, d)=\mathcal{A}_{p s}^{0,1}(r, d) / \mathcal{G}^{\mathbb{C}}
$$

Respectively, define the moduli space of stable vector bundles as the quotient

$$
\mathcal{N}_{s}(r, d)=\mathcal{A}_{s}^{0,1}(r, d) / \mathcal{G}^{\mathbb{C}} \subseteq \mathcal{N}(r, d)
$$

Remark 5. The quotient $\mathcal{A}^{0,1}(r, d) / \mathcal{G}^{\mathbb{C}}$ is not Hausdorff so, we may lose a lot of interesting properties.

Narasimhan and Seshadri [14] charaterize this variety (see also Atiyah and Bott [1] ), and conclude in their work that:

Theorem 1. [14] If $\operatorname{GCD}(r, d)=1$, then $\mathcal{A}_{p s}^{0,1}=\mathcal{A}_{s}^{0,1}$ and $\mathcal{N}(r, d)=\mathcal{N}_{s}(r, d)$ is a smooth complex projective variety of dimension

$$
\operatorname{dim}_{\mathbb{C}}(\mathcal{N}(r, d))=r^{2}(g-1)+1
$$

\subsection{Hitchin construction}

Hitchin [9] works with the Yang-Mills self-duality equations (SDE)

$$
\left\{\begin{array}{c}
F_{A}+\left[\varphi, \varphi^{*}\right]=0 \\
\bar{\partial}_{A} \varphi=0
\end{array}\right.
$$

where $\varphi \in \Omega^{1,0}(\Sigma, \operatorname{End}(\mathcal{E}))$ is a complex auxiliary field and $F_{A}$ is the curvature of a connection $d_{A}$ which is compatible with the holomorphic structure of the bundle $E=\left(\mathcal{E}, \bar{\partial}_{A}\right)$, and $\mathcal{E}$ has $\operatorname{rank} \operatorname{rk}(\mathcal{E})=2$ and degree $\operatorname{deg}(\mathcal{E})=1$. Hitchin calls $\varphi$ Higgs field, because it shares a lot of the physical and gauge properties of those of the Higgs boson. Here, $\varphi^{*}$ denotes the adjoint of $\varphi$ with respect to the hermitian metric on $E$, and $[\cdot, \cdot]$ denotes the extension of the Lie bracket to Lie algebra-valued forms. For details, the reader may see Hitchin [9]. 
The set of solutions

$$
\beta(\mathcal{E}):=\left\{\left(\bar{\partial}_{A}, \varphi\right) \mid \quad \text { solution of } \quad(2)\right\} \subseteq \mathcal{A}^{0,1}(\mathcal{E}) \times \Omega^{1,0}(\Sigma, \operatorname{End}(\mathcal{E}))
$$

and the collection

$$
\beta_{p s}(2,1):=\{\beta(\mathcal{E}) \mid \mathcal{E} \quad \text { polystable, } \quad \operatorname{rk}(\mathcal{E})=2, \operatorname{deg}(\mathcal{E})=1\},
$$

allow Hitchin to construct the Moduli space of solutions to SDE (2)

$$
\mathcal{M}^{Y M}(2,1)=\beta_{p s}(2,1) / \mathcal{G}^{\mathbb{C}}
$$

and

$$
\mathcal{M}_{s}^{Y M}(2,1)=\beta_{s}(2,1) / \mathcal{G}^{\mathbb{C}} \subseteq \mathcal{M}^{Y M}(2,1),
$$

the moduli space of stable solutions to $\operatorname{SDE}(2)$.

Remark 6. Since $\operatorname{GCD}(2,1)=1$, then $\mathcal{A}_{p s}^{0,1}=\mathcal{A}_{s}^{0,1}$ and so

$$
\mathcal{M}^{Y M}(r, d)=\mathcal{M}_{s}^{Y M}(r, d) .
$$

An alternative algebro-geometric construction is presented also in the work of Hitchin [9] [10]:

Definition 10. A Higgs bundle over $\Sigma$ is a pair $(E, \varphi)$ where $E \rightarrow \Sigma$ is a holomorphic vector bundle together with $\varphi$, an endomorphism of $E$ twisted by $K=T^{*}(\Sigma) \rightarrow \Sigma$, the canonical line bundle of the surface $\Sigma: \varphi: E \rightarrow E \otimes K$. The field $\varphi$ is what Hitchin calls Higgs field.

There is a condition of stability analogous to the one for vector bundles, but with reference just to subbundles preserved by the endomorphism $\varphi$ :

Definition 11. A subbundle $F \subseteq E$ is said to be $\varphi$-invariant if $\varphi(F) \subseteq F \otimes K$. A Higgs bundle is said to be semistable [respectively, stable] if $\mu(F) \leqslant \mu(E)$ [resp., $\mu(F)<\mu(E)$ ] for any nonzero $\varphi$-invariant subbundle $F \subseteq E$ [resp., $F \subsetneq E]$. Finally, $(E, \varphi)$ is called polystable if it is the direct sum of stable $\varphi$-invariant subbundles, all of the same slope.

With this notion of stability in mind, Hitchin [9] constructs the moduli space of Higgs bundles as the quotient

$$
\mathcal{M}^{H}(2,1)=\{(E, \varphi) \mid E \text { polystable }\} / \mathcal{G}^{\mathbb{C}}
$$

and the subspace

$$
\mathcal{M}_{s}^{H}(2,1)=\{(E, \varphi) \mid E \quad \text { stable } \quad\} / \mathcal{G}^{\mathbb{C}} \subseteq \mathcal{M}^{H}(2,1),
$$

of stable Higgs bundles. 
Remark 7. Again, $\operatorname{GCD}(2,1)=1$ implies $\mathcal{M}^{H}(2,1)=\mathcal{M}_{s}^{H}(2,1)$.

Finally, Hitchin [9] concludes:

Theorem 2. [9] There is a homeomorphism of topological spaces

$$
\mathcal{M}^{H}(2,1) \cong \mathcal{M}^{Y M}(2,1) .
$$

Hitchin [9] computes the real dimension of the moduli space of stable rank two pairs $(E, \varphi), \mathcal{M}(2,1)$ for brief:

Theorem 3. [9, Th. 5.8.] Let $\Sigma=\Sigma_{g}$ be a compact Riemann surface of genus $g>1$. The moduli space $\mathcal{M}(2,1)$ of all stable pairs $(E, \varphi)$, where $E \rightarrow \Sigma$ is a rank two holomorphic vector bundle of degree one, and $\varphi$ is a trace free holomorphic section of $\operatorname{End}(E) \otimes K$, is a smooth real manifold of dimension

$$
\operatorname{dim}_{\mathbb{R}}(\mathcal{M}(2,1))=12(g-1) .
$$

Corollary 1. The space $\mathcal{M}(2,1)$ is a quasi-projective variety of complex dimension

$$
\operatorname{dim}_{\mathbb{C}}(\mathcal{M}(2,1))=3(2 g-2) .
$$

\subsection{General rank}

Nitsure [15] constructs the moduli space of Higgs bundles of general rank $r$ and degree $d$ using GIT, and computes its dimension:

Theorem 4. [15] The space $\mathcal{M}(r, d)$ is a quasi-projective variety of complex dimension

$$
\operatorname{dim}_{\mathbb{C}}(\mathcal{M}(r, d))=\left(r^{2}-1\right)(2 g-2) .
$$

Remark 8. Note that the result of Nitsure [15] coincides with the result of Hitchin [9] for rank two Higgs bundles.

Simpson [17] calls the pair $(E, \varphi)$ as Higgs bundle. His work contributes generalizing Higgs bundles to higher dimensions and proving an analogous proposition to Theorem 3 for general rank, with the same notion of stability in mind, considering the moduli space of Higgs bundles as the quotient

$$
\mathcal{M}^{H}(r, d)=\{(E, \varphi) \mid E \text { polystable }\} / \mathcal{G}^{\mathbb{C}}
$$

and the subspace

$$
\mathcal{M}_{s}^{H}(r, d)=\{(E, \varphi) \mid E \quad \text { stable } \quad\} / \mathcal{G}^{\mathbb{C}} \subseteq \mathcal{M}^{H}(r, d),
$$

of stable Higgs bundles. 
Remark 9. Once again, $\operatorname{GCD}(r, d)=1$ implies $\mathcal{M}^{H}(r, d)=\mathcal{M}_{s}^{H}(r, d)$. See Simpson [17] for details.

Theorem 5. [17, Prop. 1.5] There is a homeomorphism of topological spaces

$$
\mathcal{M}^{H}(r, d) \cong \mathcal{M}^{Y M}(r, d)
$$

\section{Stratifications}

\subsection{Harder-Narasimhan filtrations and the Shatz stratification}

The Harder-Narasimhan filtration of a vector bundle was introduced in [7] and studied systematically by Shatz [16]. It plays an important role in the work of Atiyah and Bott [1]. We refer the reader to these references for details on what follows.

Let $E$ be a holomorphic vector bundle on $X$. A Harder-Narasimhan Filtration of $E$, is a filtration of the form

$$
\operatorname{HNF}(E): E=E_{s} \supset E_{s-1} \supset \cdots \supset E_{1} \supset E_{0}=0,
$$

which satisfies the following two properties:

(i) $\mu\left(E_{j+1} / E_{j}\right)<\mu\left(E_{j} / E_{j-1}\right)$ for $1 \leqslant j \leqslant s-1$.

(ii) $E_{j} / E_{j-1}$ is semistable for $1 \leqslant j \leqslant s$.

For brevity, when we have a filtration $E=E_{s} \supset E_{s-1} \supset \cdots \supset E_{1} \supset E_{0}=0$ we shall sometimes write $\bar{E}_{j}=E_{j} / E_{j-1}$ for the subquotients. The associated graded vector bundle is

$$
\operatorname{Gr}(E)=\bigoplus_{j=1}^{s} E_{j} / E_{j-1}=\bigoplus_{j=1}^{s} \bar{E}_{j}
$$

Remark 10. Property (i) $\mu\left(\bar{E}_{j+1}\right)<\mu\left(\bar{E}_{j}\right)$ for $1 \leqslant j \leqslant s-1$ is equivalent to $\mu\left(E_{j+1}\right)<\mu\left(E_{j}\right)$ for $1 \leqslant j \leqslant s-1$, which is intuitively clear if we take a view to the Harder-Narasimhan Polygon (for more details, see Shatz [16, Prop. 5]), Figure 1. 


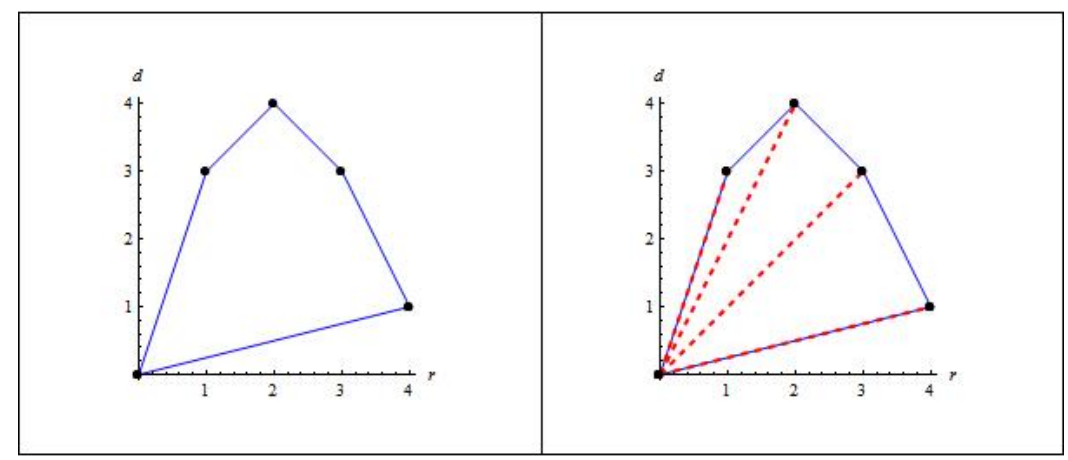

Figure 1: On the left, the Harder-Narasimhan Polygon, where the black points represent the pairs $\left(r_{i}, d_{i}\right)$ for $E_{i}$, and the blue line segments represent segments with slope $\mu\left(V_{i}\right)=\mu\left(E_{i} / E_{i-1}\right)$. On the right, the red-dashed line segments represent segments with slope $\mu\left(E_{i}\right)$.

Any vector bundle $E$ has a unique Harder-Narasimhan filtration. The HarderNarasimhan polygon is the polygon in the first quadrant of the $(r, d)$-plane with vertices $\left(\operatorname{rk}\left(E_{j}\right), \operatorname{deg}\left(E_{j}\right)\right)$ for $j=0, \ldots, s$. The slope of the line joining $\left(\operatorname{rk}\left(E_{j-1}\right), \operatorname{deg}\left(E_{j-1}\right)\right)$ and $\left(\operatorname{rk}\left(E_{j}\right), \operatorname{deg}\left(E_{j}\right)\right)$ is $\mu\left(\bar{E}_{j}\right)$. Condition (i) above says that the Harder-Narasimhan polygon is convex. Clearly this is equivalent to saying that $\mu\left(E_{j}\right)<\mu\left(E_{j-1}\right)$ for $j=2, \ldots, s$.

The Harder-Narasimhan type of $E$ is the vector in $\mathbb{Q}^{r}$ :

$$
\operatorname{HNT}(E)=\vec{\mu}=\left(\mu\left(\bar{E}_{1}\right), \ldots, \mu\left(\bar{E}_{1}\right), \ldots, \mu\left(\bar{E}_{s}\right), \ldots, \mu\left(\bar{E}_{s}\right)\right)
$$

where the slope of each $\bar{E}_{j}$ is repeated $\operatorname{rk}\left(\bar{E}_{j}\right)$ times.

As a consequence of Shatz [16, Prop. $10 \& 11]$, there is a finite stratification of $\mathcal{M}(r, d)$ by the Harder-Narasimhan type of the underlying vector bundle $E$ of a Higgs bundle $(E, \varphi)$ :

$$
\mathcal{M}(r, d)=\bigcup_{\mu} U_{\mu}^{\prime}
$$

where $U_{\mu}^{\prime} \subseteq \mathcal{M}(r, d)$ is the subspace of Higgs bundles $(E, \varphi)$ whose underlying vector bundle $E$ has Harder-Narasimhan type $\mu$. This stratification is known as the Shatz stratification. Note that there is an open dense stratum corresponding to Higgs bundles $(E, \varphi)$ for which the underlying vector bundle $E$ is itself stable. Since $\varphi \in H^{0}(\operatorname{End}(E) \otimes K) \cong H^{1}(\operatorname{End}(E))^{*}$ (by Serre duality) such a Higgs bundle represents a point in the cotangent bundle of the moduli space of stable bundles $\mathcal{N}(r, d)$. In other words,

$$
U_{(d / r, \ldots, d / r)}^{\prime}=T^{*} \mathcal{N}(r, d) \subseteq \mathcal{M}(r, d) .
$$




\subsection{The $C^{*}$-action and the Białynicki-Birula stratification}

We review some standard facts about the $\mathbb{C}^{*}$-action on $\mathcal{M}(r, d)$. For more details see, e.g., Simpson [17].

The holomorphic action of the multiplicative group $\mathbb{C}^{*}$ on $\mathcal{M}(r, d)$ is defined by multiplication $z \cdot(E, \varphi) \mapsto(E, z \cdot \varphi)$. The limit $\left(E_{0}, \varphi_{0}\right)=\lim _{z \rightarrow 0} z \cdot(E, \varphi)$ exists for all $(E, \varphi) \in \mathcal{M}(r, d)$. Moreover, this limit is fixed by the $\mathbb{C}^{*}$-action. A Higgs bundle $(E, \varphi)$ is a fixed point of the $\mathbb{C}^{*}$-action if and only if it is a Hodge bundle: there is a decomposition $E=\bigoplus_{j=1}^{p} E_{j}$ with respect to which the Higgs field $\varphi$ has weight one: $\varphi: E_{j} \rightarrow E_{j+1} \otimes K$. The type of the Hodge bundle $(E, \varphi)$ is $\left(\operatorname{rk}\left(E_{1}\right), \ldots, \operatorname{rk}\left(E_{p}\right)\right)$. Let $\left\{F_{\lambda}\right\}$ be the irreducible components of the fixed point locus of $\mathbb{C}^{*}$ on $\mathcal{M}(r, d)$. Consider the upper flow sets

$$
U_{\lambda}^{+}:=\left\{(E, \varphi) \in \mathcal{M}: \lim _{z \rightarrow 0} z \cdot(E, \varphi) \in F_{\lambda}\right\} .
$$

Then we have the Biatynicki-Birula stratification (cf. [2]) of $\mathcal{M}(r, d)$ :

$$
\mathcal{M}=\bigcup_{\lambda} U_{\lambda}^{+}
$$

\section{Recent results}

\subsection{Stratifications}

Motivated by the results of Hausel [8], in terms of stratifications of the moduli space of rank two Higgs bundles, we have tried to improve the results of Hausel to rank three. So far, here are the main results. Recall that here, we only announce the results, no formal proofs are given. The reader can find the detailed proofs in [5]. First, we will present the bounds on Harder-Narasimhan types for rank three Higgs bundles. Let $(E, \varphi)$ be a rank 3 Higgs bundle. Let $\left(\mu_{1}, \mu_{2}, \mu_{3}\right)$ be the Harder-Narasimhan type of $E$, so that $\mu_{1} \geqslant \mu_{2} \geqslant \mu_{3}$ and $\mu_{1}+\mu_{2}+\mu_{3}=3 \mu$, where $\mu=\mu(E)$. We can write the Harder-Narasimhan filtration of the vector bundle $E$ as follows:

$$
\operatorname{HNF}(E): 0=E_{0} \subseteq E_{1} \subseteq E_{2} \subseteq E_{3}=E,
$$

where we have made the convention that $E_{i}=E_{j}$ if $\mu_{i}=\mu_{j}$. Thus, for example, if $\mu_{1}=\mu_{2}>\mu_{3}$ then the Harder-Narasimhan filtration is

$$
\operatorname{HNF}(E): 0=E_{0} \subseteq E_{1}=E_{2} \subseteq E_{3}=E
$$


and $\operatorname{rk}\left(E_{1}\right)=\operatorname{rk}\left(E_{2}\right)=2$. Similarly, if $\mu_{1}>\mu_{2}=\mu_{3}$ then $\operatorname{rk}\left(E_{1}\right)=1$ and $\operatorname{rk}\left(E_{2}\right)=3$.

Remark 11. Let $(E, \varphi)$ be a stable Higgs bundle such that $E$ is an unstable vector bundle of $\operatorname{HNT}(E)=\left(\mu_{1}, \mu_{2}, \mu_{3}\right)$. Then $E_{1} \subseteq E_{2}$ is destabilizing and hence, by stability of $(E, \varphi)$, we have $\varphi_{21} \neq 0$ where $\varphi=\left(\begin{array}{ccc}0 & 0 & 0 \\ \varphi_{21} & 0 & 0 \\ 0 & \varphi_{32} & 0\end{array}\right)$. Similarly $E_{2} \subseteq E$ is destabilizing and so $\varphi_{32} \neq 0$ (unless $\mu_{2}=\mu_{3} \Longleftrightarrow E_{2}=E$ ).

After analyzing the image and the kernel of $\varphi$, we get the following bounds:

Proposition 1. Let $(E, \varphi)$ be a semistable rank 3 Higgs bundle of $\operatorname{HNT}(E)=$ $\left(\mu_{1}, \mu_{2}, \mu_{3}\right)$. Then

$$
\begin{aligned}
& 0 \leqslant \mu_{1}-\mu_{2} \leqslant 2 g-2, \\
& 0 \leqslant \mu_{2}-\mu_{3} \leqslant 2 g-2 .
\end{aligned}
$$

The purpose now will be to analyze the $\mathbb{C}^{*}$-action limit as $z \rightarrow 0$ of $z \cdot(E, \varphi)$ as a function of $\operatorname{HNT}(E)$. Let start by trivial filtrations. Let $(E, \varphi)$ be a stable Higgs bundle. When the underlying vector bundle $E$ is itself stable, clearly $\lim _{z \rightarrow 0} z \cdot(E, \varphi)=(E, 0)$. Hence we have the following result, valid for any rank.

Proposition 2. Let $(E, \varphi) \in \mathcal{M}(r, d)$. Then $\lim _{z \rightarrow 0} z \cdot(E, \varphi)=(E, 0)$ if and only if $E$ is stable.

Proof. It only remains to observe that if $(E, 0)=\lim _{z \rightarrow 0} z \cdot(E, \varphi)$ is a stable Higgs bundle then $E$ is a stable vector bundle.

Now, let see non-trivial Harder-Narasimhan filtrations. Let consider rank 3 stable Higgs bundles $(E, \varphi)$ and assume that $(3, d)=1$.

Theorem 6. Let $(E, \varphi) \in \mathcal{M}(3, d)$ be such that $E$ is an unstable vector bundle of slope $\mu$ and $\operatorname{HNT}(E)=\left(\mu_{1}, \mu_{2}, \mu_{3}\right)$. Then the limit $\left(E_{0}, \varphi_{0}\right)=\lim _{z \rightarrow 0}(E, z \cdot \varphi)$ is given as follows.

(1) Assume that $\mu_{2}<\mu$. Then $\mu_{1}>\mu_{2} \geqslant \mu_{3}$ and one of the following alternatives holds.

(1.1) The slope of $I=\varphi_{21}\left(E_{1}\right) \otimes K^{-1}$ satisfies $\mu_{1}-(2 g-2) \leqslant \mu(I)<$ $-\frac{1}{3} \mu_{1}+\frac{2}{3} \mu_{2}+\frac{2}{3} \mu_{3}$ and $\left(E_{0}, \varphi_{0}\right)$ is the following Hodge bundle of type $(1,2)$ :

$$
\left(E_{0}, \varphi_{0}\right)=\left(E_{1} \oplus E / E_{1},\left(\begin{array}{cc}
0 & 0 \\
\varphi_{21} & 0
\end{array}\right)\right) .
$$

The associated graded vector bundle is $\operatorname{Gr}\left(E_{0}\right)=\operatorname{Gr}(E)$. 
(1.2) The slope of $I=\varphi_{21}\left(E_{1}\right) \otimes K^{-1}$ satisfies $-\frac{1}{3} \mu_{1}+\frac{2}{3} \mu_{2}+\frac{2}{3} \mu_{3}<$ $\mu(I) \leqslant \mu_{3}$ and $\left(E_{0}, \varphi_{0}\right)$ is the following Hodge bundle of type $(1,1,1)$ :

$$
\left(E_{0}, \varphi_{0}\right)=\left(E_{1} \oplus I \oplus\left(E / E_{1}\right) / I,\left(\begin{array}{ccc}
0 & 0 & 0 \\
\varphi_{21} & 0 & 0 \\
0 & \varphi_{32} & 0
\end{array}\right)\right),
$$

where $\varphi_{21}$ and $\varphi_{32}$ are induced from $\varphi$. The associated graded vector bundle is $\operatorname{Gr}\left(E_{0}\right)=E_{1} \oplus\left(E / E_{1}\right) / I \oplus I$ and $\operatorname{HNT}\left(E_{0}\right)=$ $\left(\mu_{1}, \mu_{2}+\mu_{3}-\mu(I), \mu(I)\right)$.

(1.3) The slope of $I=\varphi_{21}\left(E_{1}\right) \otimes K^{-1}$ satisfies $\mu(I)=\mu_{2}$ and the strict inequality $\mu_{3}<\mu_{2}$ holds. Moreover, the line bundle $I=E_{2} / E_{1}$ and $\left(E_{0}, \varphi_{0}\right)$ is the following Hodge bundle of type $(1,1,1)$ :

$$
\left(E_{0}, \varphi_{0}\right)=\left(E_{1} \oplus E_{2} / E_{1} \oplus E / E_{2},\left(\begin{array}{ccc}
0 & 0 & 0 \\
\varphi_{21} & 0 & 0 \\
0 & \varphi_{32} & 0
\end{array}\right)\right),
$$

where $\varphi_{32}$ is induced from $\varphi$. The associated graded vector bundle is $\operatorname{Gr}\left(E_{0}\right)=\operatorname{Gr}(E)$.

(2) Suppose that $\mu_{2}>\mu$. Then $\mu_{1} \geqslant \mu_{2}>\mu_{3}$ and one of the following alternatives holds.

(2.1) The slope of $N=\operatorname{ker}\left(\varphi_{32}\right)$ satisfies $\mu_{1}+\mu_{2}-\mu_{3}-(2 g-2) \leqslant$ $\mu(N)<\mu$ and $\left(E_{0}, \varphi_{0}\right)$ is the following Hodge bundle of type $(2,1)$ :

$$
\left(E_{0}, \varphi_{0}\right)=\left(E_{2} \oplus E / E_{2},\left(\begin{array}{cc}
0 & 0 \\
\varphi_{32} & 0
\end{array}\right)\right) .
$$

The associated graded vector bundle is $\operatorname{Gr}\left(E_{0}\right)=\operatorname{Gr}(E)$.

(2.2) The slope of $N=\operatorname{ker}\left(\varphi_{32}\right)$ satisfies $\mu<\mu(N) \leqslant \mu_{2}$ and $\left(E_{0}, \varphi_{0}\right)$ is the following Hodge bundle of type $(1,1,1)$ :

$$
\left(E_{0}, \varphi_{0}\right)=\left(N \oplus E_{2} / N \oplus E / E_{2},\left(\begin{array}{ccc}
0 & 0 & 0 \\
\varphi_{21} & 0 & 0 \\
0 & \varphi_{32} & 0
\end{array}\right)\right),
$$

where $\varphi_{21}$ and $\varphi_{32}$ are induced from $\varphi$. The associated graded vector bundle is $\operatorname{Gr}\left(E_{0}\right)=E_{2} / N \oplus N \oplus E / E_{2}$ and $\operatorname{HNT}\left(E_{0}\right)=$ $\left(\mu_{1}+\mu_{2}-\mu(N), \mu(N), \mu_{3}\right)$.

(2.3) The slope of $N=\operatorname{ker}\left(\varphi_{32}\right)$ satisfies $\mu(N)=\mu_{1}$ and the strict inequality $\mu_{1}>\mu_{2}$ holds. Moreover the line bundle $N=E_{1}$ and $\left(E_{0}, \varphi_{0}\right)$ is the following Hodge bundle of type $(1,1,1)$ :

$$
\left(E_{0}, \varphi_{0}\right)=\left(E_{1} \oplus E_{2} / E_{1} \oplus E / E_{2},\left(\begin{array}{ccc}
0 & 0 & 0 \\
\varphi_{21} & 0 & 0 \\
0 & \varphi_{32} & 0
\end{array}\right)\right),
$$

where $\varphi_{21}$ and $\varphi_{32}$ are induced from $\varphi$. The associated graded vector bundle is $\operatorname{Gr}\left(E_{0}\right)=\operatorname{Gr}(E)$. 
Remark 12. Strictly speaking, we should use the saturated bundle of the sheaf $I=\varphi_{21}\left(E_{1}\right) \otimes K^{-1}$ as in [5]. We abuse of notation here.

Corollary 2. Let $(E, \varphi) \in \mathcal{M}(3, d)$ be such that $E$ is an unstable vector bundle of slope $\mu$ and $\operatorname{HNT}(E)=\left(\mu_{1}, \mu_{2}, \mu_{3}\right)$. Assume that $\mu_{1}-\mu_{3}>2 g-2$. Then the limit $\left(E_{0}, \varphi_{0}\right)=\lim _{z \rightarrow 0}(E, z \cdot \varphi)$ is given by (1.3) of Theorem 6 if $\mu_{2}<\mu$, and by (2.3) of Theorem 6 if $\mu_{2}>\mu$.

Corollary 3. Let $\left(E_{0}=L_{1} \oplus L_{2} \oplus L_{3}, \varphi_{0}=\left(\begin{array}{ccc}0 & 0 & 0 \\ \varphi_{21} & 0 & 0 \\ 0 & \varphi_{32} & 0\end{array}\right)\right)$ be a Hodge bundle of type $(1,1,1)$ with $\mu\left(L_{1}\right)-\mu\left(L_{3}\right)>2 g-2$. Then $\mu\left(L_{1}\right)>\mu\left(L_{2}\right)>\mu\left(L_{3}\right)$ and any $(E, \varphi)$ such that $\lim _{z \rightarrow 0}(E, z \cdot \varphi)=\left(E_{0}, \varphi_{0}\right)$ satisfies $\operatorname{Gr}(E)=\operatorname{Gr}\left(E_{0}\right)$.

Proof. Since $\varphi_{21} \neq 0$, we have $\mu\left(L_{2}\right)+2 g-2 \geqslant \mu\left(L_{1}\right)$. This, together with $\mu\left(L_{1}\right)-\mu\left(L_{3}\right)>2 g-2$ implies that $\mu\left(L_{2}\right)>\mu\left(L_{3}\right)$. Since in Cases (1.2) and (2.2) of Theorem 6 one has $\mu\left(L_{2}\right) \leqslant \mu\left(L_{3}\right)$, it follows that the limit $\left(E_{0}, \varphi_{=}\right)$ must arise either from Case (1.3) or from Case (2.3). The conclusion follows since in these cases $\operatorname{Gr}(E)=\operatorname{Gr}\left(E_{0}\right)$.

The two previous corollaries lead to an identification between Shatz strata and Białynicki-Birula strata in some cases. Recall that the set $U_{\left(l_{1}, l_{2}, l_{3}\right)}^{+}$denotes the Białynicki-Birula stratum of Higgs bundles whose limits lie in $F_{\left(l_{1}, l_{2}, l_{3}\right)}$ and that $U_{\left(l_{1}, l_{2}, l_{3}\right)}^{\prime}$ denotes the Shatz stratum of Higgs bundles whose HarderNarasimhan type is $\left(l_{1}, l_{2}, l_{3}\right)$.

Theorem 7. Let $\left(l_{1}, l_{2}, l_{3}\right)$ be such that $l_{1}-l_{3}>2 g-2$. Then the corresponding Shatz and Biatynicki-Birula strata in $\mathcal{M}(3, d)$ coincide:

$$
U_{\left(l_{1}, l_{2}, l_{3}\right)}^{\prime}=U_{\left(l_{1}, l_{2}, l_{3}\right)}^{+}
$$

\subsection{Homotopy}

If we consider a fixed point $p \in X$ as a divisor $p \in \operatorname{Sym}^{1}(X)=X$, and $L_{p}$ the line bundle that corresponds to that divisor $p$, we get a complex of the form

$$
E \stackrel{\varphi^{k}}{\longrightarrow} E \otimes K \otimes L_{p}^{\otimes k},
$$

where $\varphi^{k} \in H^{0}\left(X, \operatorname{End}(E) \otimes K \otimes L_{p}^{\otimes k}\right)$ is a Higgs field with poles of order $k$. So, we call such a complex as a $k$-Higgs bundle and $\varphi^{k}$ as its $k$-Higgs field. As well as for a Higgs bundle, a $k$-Higgs bundle $\left(E, \varphi^{k}\right)$ is stable (respectively semistable) if the slope of any $\varphi^{k}$-invariant subbundle of $E$ is strictly less (respectively less or equal) than the slope of $E: \mu(E)$. Finally, $\left(E, \varphi^{k}\right)$ is called 
polystable if $E$ is the direct sum of stable $\varphi^{k}$-invariant subbundles, all of the same slope. Motivated by the results of Bradlow, García-Prada and Gothen [4], and once again by the work of Hausel [8], in terms of the homotopy groups of the moduli space of Higgs bundles and $k$-Higgs bundles respectively, we have tried to improve the result of Hausel to higher rank. So far, here is one of the main results. Recall that here, we only announce the result, no proof is given. The reader can find the full detailed proof in [20].

Theorem 8. [20, Thm. 1.1] Suppose the rank is either $r=2$ or $r=3$, and $\operatorname{GCD}(r, d)=1$. Then, for all $n$ exists $k_{0}$, depending on $n$, such that

$$
\pi_{j}\left(\mathcal{M}_{\Lambda}^{k}(r, d)\right) \stackrel{\cong}{\longrightarrow} \pi_{j}\left(\mathcal{M}_{\Lambda}^{\infty}(r, d)\right)
$$

for all $k \geqslant k_{0}$ and for all $j \leqslant n-1$.

\subsection{Further research}

We are currently working on interesting results related with the homotopy of the moduli space of Higgs bundles, in terms of stratifications in the nilpotent cone [6], and in terms of Morse Theory and Variations of Hodge Structures [21, 20]. We encourage the reader to explore those results in the references above mentioned.

\section{Acknowledgements}

We thank Peter B. Gothen for introducing us to the beautiful subject of Higgs bundles. We thank the referees for their comments and suggestions. We thank organizers of XXI-SIMMAC-2018 for the opportunity of share our work with the scientific community. Financial support from Vicerrectoría de Investigación, Universidad de Costa Rica, is acknowledged.

Research supported by Universidad de Costa Rica through Escuela de Matemática and through CIMM (Centro de Investigaciones Matemáticas y Metamatemáticas), Project 820-B8-224. This work is partly based on the Ph.D. Project [19] called "Homotopy Groups of the Moduli Space of Higgs Bundles", supported by FEDER through Programa Operacional Factores de Competitividade-COMPETE, and also supported by FCT (Fundação para a Ciência e a Tecnologia) through the projects PTDC/MAT-GEO/0675/2012 and PEstC/MAT/UI0144/2013 with grant reference SFRH/BD/51174/2010. 


\section{References}

[1] M.F. Atiyah, R. Bott, The Yang-Mills equations over Riemann surfaces, Phil. Trans. R. Soc. Lond. 308 (1982), no. 1505, 523-615.

[2] A. Białynicki-Birula, Some theorems on actions of algebraic groups, Ann. of Math. 98 (1973), 480-497.

[3] S.B. Bradlow, O. García-Prada, P.B. Gothen, What is a Higgs bundle?, Notices of the American Mathematical Society 54 (2007), no. 8, 980-981.

[4] S.B. Bradlow, O. García-Prada, P.B. Gothen, Homotopy groups of moduli spaces of representations, Topology 47 (2008), no. 4, 203-224.

[5] P.B. Gothen, R.A. Zúñiga-Rojas, Stratifications on the moduli space of Higgs bundles, Portugaliae Mathematica 74 (2017), 127-148.

[6] P.B. Gothen, R.A. Zúñiga-Rojas, Stratifications on the Nilpotent Cone of the Hitchin Map, in progress.

[7] G. Harder, M.S. Narasimhan, On the Cohomology Groups of Moduli Spaces of Vector Bundles on Curves, Math. Ann. 212 (1975), 215-248.

[8] T. Hausel, Geometry of the moduli space of Higgs bundles, Ph.D. Thesis, Univ. of Cambridge, 1998.

[9] N.J. Hitchin, The self-duality equations on a Riemann surface, Proc. London Math. Soc. 55 (1987), no. 3, 59-126.

[10] N.J. Hitchin, Gauge theory on Riemann surfaces (M. Carvalho, X. GomezMont and A. Verjovsky, editors), Lectures on Riemann surfaces: proceedings of the college on Riemann surfaces, Italy, 99-118, 1989.

[11] S. Kobayashi, Differential geometry of complex vector bundles, Publications of the Mathematical Society of Japan, Vol. 15, Iwanami Shoten, Publishers and Princeton Univ. Press, 1987.

[12] M. Lübke, A. Teleman, The Kobayashi-Hitchin Correspondence, World Scientific Publishing Co., 1995.

[13] D. Mumford, J. Fogarty, F. Kirwan, Geometric Invariant Theory, Springer, 1994. 
[14] M.S. Narasimhan, C.S. Seshadri, Stable and unitary vector bundles on a compact Riemann surface, Annals of Mathematics, Second Series, Vol. 82, No. 3 (Nov., 1965), 540-567.

[15] N. Nitsure, Moduli space of semistable pairs on a curve, Proc. London Math. Soc. Vol. s3-62, Issue 2 (March, 1991), 275-300.

[16] S.S. Shatz, The Decomposition and Specialization of Algebraic Families of Vector Bundles, Compositio Mathematica 35 (1977), no. 2., 163-187.

[17] C.T. Simpson, Higgs bundles and local systems, Publ. Math. de l'IHÉS, Tome 75, (1992) 5-95.

[18] C.N. Yang, R.L. Mills, Conservation of isotopic spin and isotopic gauge invariance, Phys. Rev. 96 (1954), no. 1, 191-195.

[19] R.A. Zúñiga-Rojas, Homotopy groups of the moduli space of Higgs bundles, Ph.D. Thesis, Universidade do Porto, 2015.

[20] R.A. Zúniga-Rojas, Stabilization of the homotopy groups of the moduli spaces of k-Higgs bundles, Revista Colombiana de Matemáticas 52 (2018), no. 1, 9-31.

[21] R.A. Zúniga-Rojas, Variations of Hodge structures of rank three k-Higgs bundles. Preprint, available at arXiv:1803.01936v3 [math.AG]. 